\title{
Tabu Search Algorithm for RP Selection in PIM-SM Multicast Routing
}

\author{
HUA WANG, MIN ZHANG, JUN MA \\ School of Computer Science and Technology \\ Shandong University \\ No.73 JingShi Road, Jinan, Shandong Province \\ CHINA
}

\begin{abstract}
To construct a multicast tree is the basis of multicast data transmission. The prime problem concerning the construction of a shared multicast tree is to determine the position of the root of the shared tree, which is the center selection problem. The selection of the center directly affects the structure of the tree, and affects the performance of multicast accordingly. The center selection problem is an NPC problem. This paper proposes a multicast RP selection algorithm based on tabu search. This algorithm selects RP by considering cost and delay. Simulation results show that good performance is achieved in multicast cost, delay from end to end and register delay.
\end{abstract}

\section{Key-Words: - PIM-SM; RP Selection; Group-Based; Tabu Search; Optimization}

\section{Introduction}

As a critical technology in distributed multimedia real-time application such as teleconference, audio/vedio broadcasting, multimedia distance education and software distribution, multicast technology has been a research hotspot. To construct a multicast tree is the basis of multicast data transmission. The prime problem concerning the construction of a shared multicast tree is to determine the position of the root of the shared tree, which is the center selection problem. The center is called Core Point in CBT [1] or Rendezvous Point(RP) in PIM-SM[3].This problem is first proposed by Wall[3]. The selection of center directly affects the structure of the tree, and affects the performance of multicast accordingly. A poor selection may lead to such performance problems as high cost, high delay, high congestion. So to have effective multicast requires the selection of a good center. But the center selection problem is an NPC problem [1, 3, 4], needing a heuristic algorithm to solve. Researchers have already proposed several solutions $[3,5,6$, $7,8]$ to this problem. However, how to evaluate the merits and shortcomings of a multicast tree when a node is taken as a root and how to find a better or best center in given time and complexity has not been completely solved. The selection made is meaningless unless correct evaluation is made about the merits and shortcomings of the constructed multicast tree. Some approaches make the selection among a limited number of candidate nodes. Some search by employing greedy algorithm. This may lead to local optimization.

In this paper the center selection problem will be discussed by combining PIM-SM model. After introducing previous research findings and the basic tabu search algorithm a Tabu-search-based RP selection algorithm (TRPSA) is proposed. This proposed algorithm introduces an approach to evaluating the merits and shortcomings of the multicast tree through considering cost and delay integratedly and realizes RP selection with tabu search algorithm. Simulation results show that good performance is achieved in multicast cost, ETE (end-to-end) delay and register delay. Moreover, the algorithm had flexibility and expandability.

\section{Background}

To build a multicast tree to forward multicast datagrams is generally adopted in multicast application. The fundamental problem of multicast routing is to find a low-cost tree covering all group members. This problem is attributed to a Steiner tree problem in mathematics, which is an NPC problem. Currently multicast routing protocols are divided into Source-based Multicast Tree and Core-based Multicast Tree according to multicast tree types. The Core-based tree has better flexibility and expandability. And only routers on the tree need to retain information of group members. It performs well in the amount of state information needing to be stored in the router and the total cost of routing tree. 
But it is difficult to find the best shared tree. Researchers sometimes divide the problem into two sub-problems, i.e. center selection problem and routing selection problem.

The two frequently discussed and most representative shared trees are CBT and PIM-SM. PIM-SM is the most widely used multicast routing protocol, in which one multicast group has only one active RP. The joining and leaving of a group member is realized by hop-to-hop along the shortest path from the local router to RP with explicit Join/Leave information, with the router automatically updating the routing table. PIM-SM uses a special router called Bootstrap router (BSR), which notifies a set of candidate (viable) RPs. Every node uses Hash function to map to an RP according to the address of the group it belongs to. Unlike CBT, PIM-SM is a unidirectional shared tree, requiring that the data sent by the source node be first sent to RP, and forwarded to the receiving node by RP. Therefore, RP is a node converging traffic, responsible for forwarding data and processing the registration of source and the joining and leaving of receivers. It has heavy load. Then RP selection will, to a great extent, influence the performance of protocol. A good RP selection algorithm is needed.

\section{Existing RP selection algorithms}

Some researchers have already proposed some algorithms for RP location. One of the major differences between them lies in the node set from which to select the best as the new RP.

Ideally all the nodes in the network should be taken as candidate RPs, from which the best is obtained. This approach is called Optimal Center-Based Tree (OCBT). In reality by computing the actual cost when every node is treated as the root of a tree, the root of the tree with the lowest cost is selected as RP. It takes a large quantity of computing time.

In the Random Source-Specific Tree (RSST) heuristic, the center is randomly selected from the set of source nodes, and cannot move any more. This approach is simple, but once the selected node becomes invalid it is impossible to rebuild the multicast tree.

As for Minimal Shortest Path Tree (MSPT), it calculates the actual costs for the trees rooted at each group member, and chooses the member with the lowest cost. The problem of this approach is bigger delay of the tree. The delay bound triples that of the source-specific tree, whereas the delay bound of the core tree doubles that of the source-specific tree.
When all the nodes are group members, this approach will degenerate as OCBT.

RP selection methods can be classified into two types in terms of whether there are QoS constraints: RP selection based on QoS constraints and RP selection without QoS constraints.

\subsection{RP Selection based on QoS constraints}

This method can provide every member of the group with Multiple QoS guarantee, such as delay, jitter, bandwidth and so on. A large quantity of information such as network topology, group member, QoS and link bandwidth is needed to decide the RP of a group. QCSA (QoS-based Selection algorithm) proposed in [5] is a typical RP selection algorithm considering multi-constraints. Such algorithm is highly complex and QoS is hard to guarantee for the newly joined member.

\subsection{RP selection without QoS constraints}

Random RP selection selects any node as RP randomly without considering any network or QoS information. The advantage of this selection lies in its simplicity. But it cannot guarantee QoS at all times. The node selected may be far from group members, which may lead to higher cost, such as delay.

Selection based on topology chooses the network center as RP. Since all the RPs of the multicast groups are located at the center of the network the traffic of multicast will converge. The increase of the number of multicast groups will cause overload at RP.

Selection based on group. The distribution of group members is considered while selecting RP. The RP selected is close to the group members. Unlike the selection based on topology which selects an RP for the entire network, the selection based on group select an RP for each group, thus avoiding convergence of traffic.

An algorithm based on the source nodes of multicast is proposed in [8] to relocate an RP. It employs local search method, i.e. the node with minimal objective value is selected from all the neighboring nodes every time. If the value is smaller than that of the current node, the current node moves to this neighbor. This process is repeated until no objective value of the neighbor is smaller than the current one. This is a thought of the greedy algorithm, which may lead to local optimization and failure to reach global optimal point. The difference between [6] and [8] lies in the fact that every time from all the neighbors several nodes with minimal objective values are selected. Next time the current nodes move 
to these neighboring nodes. The terminating conditions are decided by the number of iterative times. The final solution obtained is the best solution that can be searched within iterative times. When the number of iterative times is big enough this method can guarantee the finding of global optimal solution. But a large number of useless searches exist. It has poor expandability.

\section{Basic tabu search algorithm}

Tabu search is a dynamic neighborhood search algorithm of artificial intelligence, proposed first by Glover [9]. It is the expansion of local search algorithm, which follows the neighborhood structure of local search, with the addition of a tabu table. The basic thought is to mark the obtained local optimal solution or the process of solution and avoid them in further iterative.

Tabu search algorithm manifests two strategies-centralization and diffusion, which respectively promise to reach and skip the local optimal solution. If terminating conditions are set properly, the tabu algorithm will converge to the global optimum with probability as $1[10]$.

The algorithm is described as:

STEP 1 Set a tabu list $H=\varnothing$ and select a initial solution $X_{\text {now }}$;

STEP 2 Stop computing while the conditions are met and output the result; otherwise, from the neighborhood $N\left(X_{\text {now }}\right)$ of $X_{\text {now }}$, select the candidate set $C a n_{-} N\left(X_{\text {now }}\right)$ that are untabued; from Can_N $N\left(X_{\text {now }}\right)$ select the best solution with evaluation value $X_{n \text { ext }}$. Set $X_{\text {now }}:=X_{\text {next }}$ update historical record $H$ and repeat STEP 2 ;

In the neighborhood $N\left(X_{\text {now }}\right)$ the elements that are not tabued either violate one of the Tabu conditions or hold one of the aspiration conditions.

\section{Tabu search algorithm for RP selection}

Conventional approaches based on group give up source node or receiver node, or treat source node and receiver node equally. For instance, only source nodes are considered in [8] to make the "Gathering Tree” cost minimal. RP obtained this way may make the total cost higher since it is far from receiver node. In this paper, we consider both multicast source node and receiver node and distinguish them as well.

\subsection{Evaluation of multicast tree}

To evaluate whether a node is suitable to be an RP, the usual practice is to see whether the cost is smaller when it is taken as root to form multicast tree. Some researchers estimate the cost in the best and worst cases and then average the cost to get the cost of the tree. In the best case, the tree constructed is linear in shape, i.e. all the group members are on the path from the root to the most distant member. When the distance to two members is equal it is impossible to get completely linear shape. If the number of jumps is taken as distance, then the minimal cost of tree is

$$
\text { TreeCost }_{\min }=\operatorname{MAX}(d(r p, u))+d p
$$

Where $d(r p, u)$ represents the distance from root $r p$ to group member $u, d p$ is the number of duplicate distance nodes in group. In worst cases, there is no shared link between group members and the root, asteroidal tree is formed. So

$$
\text { TreeCost }_{\max }= \begin{cases}\sum d(r p, u) \quad \text { if } \operatorname{deg}(r p)>|G| \\ \sum d(r p, u)-(|G|-\operatorname{deg}(r p)) \text { else }\end{cases}
$$

Where $\operatorname{deg}(r p)$ represent the node degree of root $r p \cdot|G|$ denotes the number of multicast group members. Finally the estimate cost of the tree is

$$
\text { TreeCost }=\left(\text { TreeCost }_{\text {min }}+\text { TreeCost }_{\max }\right) / 2
$$

The value obtained this way is the approximate value of tree cost. It is difficult to get the real cost of the tree, which makes it hard to get the best RP. An Reserved Path Algorithm RPA is proposed in [8] to compute the real cost of the tree. The thought is to view $\cos t$ of all links as a unit. The cost of the tree is represented by the total number of all the links on the tree. Nodes are defined as two states (YES/NO) on the tree to avoid the repetitive computing of shared link. That is, TreeCost $=\sum_{l \in T} \cos t(l)$

Where $T$ is the expanded tree from root to all the group members. $l$ is the link on $T$. All $\cos t(l)$ is 1. But this approach cannot reflect the real cost of the multicast tree because the data transmitted via the paths from sources to RP are different. There is no share of the edge. But the data on every path fro RP to receiver nodes are the same, and there is share. So it is necessary to distinguish these two paths. Therefore, we propose a new algorithm: the contribution of every link of the multicast tree to the cost of multicast tree is the multiplier of its cost and the actual throughput rates. Since the sending rate of every source is unknown in advance, unit measure is adopted. Then the cost of the whole multicast tree can be described as

$$
\text { TreeCost }=\sum_{u \in S} \sum_{l \in P_{p, u}} \cos t(l)+|S| \sum_{l \in S T} \cos t(l)
$$


Where $S$ is the set of source; $P_{r p, u}$ is the path from RP to node $u$; $S T$ is the set of links on the shared tree, $\cos t(l)$ is the cost of link $l$, showing the importance of the link. Formula (1) shows the amount of bandwidth occupation of the multicast tree when cost is 1 .

From the perspective of the network, it is necessary to reduce the cost of the whole multicast tree as much as possible. But from the angle of service quality sensed by the user, it is important how to minimize the waste from end to end such as delay. So while considering the cost of the tree we consider delay from end to end to evaluate the performance of the constructed multicast tree. Define the delay of the multicast tree as

$$
\begin{aligned}
\text { TreeDelay } & =|\mathrm{R}| \sum_{u \in S} \sum_{l \in P_{r, p}} \operatorname{delay}(l) \\
& +|\mathrm{S}| \sum_{v \in R} \sum_{l \in P_{r, v}} \operatorname{delay}(l)
\end{aligned}
$$

Where $S$ is the set of source, $R$ is the set of receiver nodes, $\operatorname{Delay}(l)$ is the delay of link $l$.

Since cost and delay cannot be minimal simultaneously, a tradeoff method is needed. One way is to obtain several best solutions that meet one need, and then select the best that meet the other need. The other way is to assign different weights to two needs, then obtain the weighted and minimal one. For convenience and flexibility, we use the second method. Since cost and delay are different data, with difference in data unit and amount, there needs to be some change in order that they can be added and that the sensitivity to weight can be increased. This purpose is achieved through dividing the cost by the sum of all the links' costs, and the ETE delay by the sum of all the links' delays respectively. In this way, the evaluation formula of whether a node $r p$ as the RP of certain multicast group $G(S, R)$ is proper or not is

$$
\begin{aligned}
& \operatorname{ESTV}(r p, \mathrm{G})=w \frac{\text { TreeCost }}{\sum \cos t(l)}+(1-w) \frac{\text { TreeDelay }}{\sum \operatorname{delay}(l)} \\
& =w \frac{\sum_{u \in S \in P_{p, u}} \cos t(l)+|S| \sum_{l \in S T} \cos t(l)}{\sum \cos t(l)}+ \\
& (1-w) \frac{|\mathrm{R}| \sum_{u \in S} \sum_{l \in P_{p, u}} \operatorname{delay}(l)+|\mathrm{S}| \sum_{v \in R} \sum_{l \in P_{p, v}} \operatorname{delay}(l)}{\sum \operatorname{delay}(l)}
\end{aligned}
$$

Where $\mathrm{w}$ is the tradeoff weight value between cost and delay, $w \in[0,1] . \quad w=1$ and $w=0$ correspond to the respective consideration of cost and delay only.

\subsection{TRPSA}

According to the features of RP selection, we take the solution object, i.e. node object, as tabu object; neighborhood as the set of neighboring nodes of nodes. Several parameters needed are: the maximal iterative times MIT , current best solution's maximal steady times $M S T$, candidate set maximal element number $M C$, tabu length $M L$, initial node init_ $R P$,multicast group $G(S, R)$.Steps of the algorithm are described as

/* Specifications of variables : $V(X)$ : evaluation value of solution $X ; f(X)$ : aspiration conditions of solution $X ; X_{\text {cur }}$ : current solution ; $X_{\text {curbest }}$ : current best solution ; its : iterative times ; stds : number of times of current best solution to be constant */

STEP1 Initialize tabu table $H$; letits $=s t d s=0$,

$$
\begin{aligned}
& X_{\text {cur }}=X_{\text {curbest }}=\text { init_RP } \\
& V\left(X_{\text {cur }}\right)=V\left(X_{\text {curbest }}\right)=\operatorname{ESTV}(\text { init_RP,G }) \\
& f\left(X_{\text {cur }}\right)=V\left(X_{\text {cur }}\right) ;
\end{aligned}
$$

STEP2 if its $>=M I T$ or stds $>=M S T$, return to current best solution $X_{\text {curbest }}$, end the algorithm ; otherwise continue the following steps ;

STEP3 obtain the regions of the neighborhood $N\left(X_{\text {cur }}\right)$ of the current solution $X_{\text {cur }}$;

STEP 4 Randomly take a neighbor $X$ from $N\left(X_{\text {cur }}\right)$.

If $X$ is not tabued, add it to the candidate set $\mathrm{Can}_{-} N\left(X_{\text {cur }}\right)$; if $X$ is tabued but $V(X)<f\left(X_{\text {cur }}\right)$, then aspire $X$ and add it to Can_N( $\left.X_{\text {cur }}\right)$; repeat this step until $N\left(X_{\text {cur }}\right)$ becomes empty or reach the maximal candidate number $M C$.

STEP5 if Can $N\left(X_{c u r}\right)=\varnothing$, end the algorithm and return to $X_{\text {curbest }}$; otherwise select all the nodes in Can $\_N\left(X_{\text {cur }}\right)$ and construct multicast trees with the node as root one by one and evaluate according to Formula (3);

STEP6 From Can_ $N\left(X_{\text {cur }}\right)$ select the node with the minimal estimate value and mark it as $X_{\text {min }}$. If $V\left(X_{\min }\right)>V\left(X_{\text {cur }}\right)$, let $f\left(X_{\text {min }}\right)=f\left(X_{\text {cur }}\right)$, otherwise set $f\left(X_{\min }\right)=V\left(X_{\min }\right) \quad$; if $V\left(X_{\min }\right)<V\left(X_{\text {cur }}\right)$, then set $X_{\text {curbest }}=X_{\min }$, and reset stds $=0$;

STEP7 tabu $X_{\text {cur }}$, let $X_{\text {cur }}=X_{\text {min }}$; update the tabu table $H$; increase 1 to its, stds respectively , go to STEP 2 ;

The complexity of this algorithm lies in that when evaluating nodes multicast tree needs to be 
constructed. The complexity degree to evaluate the algorithm is $O\left(|G| * n^{2}+|E|\right)=O\left(|G| * n^{2}\right)$, of which $|G|$ is the number of group members and $|E|$ is the number of edges in the network. To avoid evaluating the same node many times, the nodes evaluated is recorded while realizing the algorithm. The total complexity of the algorithm in the worst case is $O\left(|G| * n^{3}\right)$.

\section{Simulation results and analysis}

To examine the performance of this algorithm, we compare it with Random, Topology Center (TC) and (Weight Center) WC algorithms through simulation.

We define topology center and weight-center as: In network topology, the maximum distance between one node and all the rest nodes is called the eccentricity distance in this network. If the number of jumps of paths between nodes is taken as distance, then the node with the minimal eccentricity distance in the network is called the topology center. If the weighted sum of the edge of paths between nodes is taken as distance, then the node with the minimal eccentricity distance in the network is called as the topology weight-center.

All network topologies used in our simulation are generated via Waxman topology model [11]( $\alpha=0.25, \beta=0.4)$. There are ten group members, who are assigned randomly in the network. All simulation results are obtained by using $\mathrm{VC}++$ 6.0. In simulations, the cost of multicast tree, average ETE delay and average register delay are computed. The cost of multicast tree is shown in the sum of average throughput rate of all the edges in the network (the cost of edge is measured in unit). ETE delay refers to the time delay from source node to receiver node. Register delay refers to the time interval lasting from sending the request of joining group to beginning to receive data packet from the group.

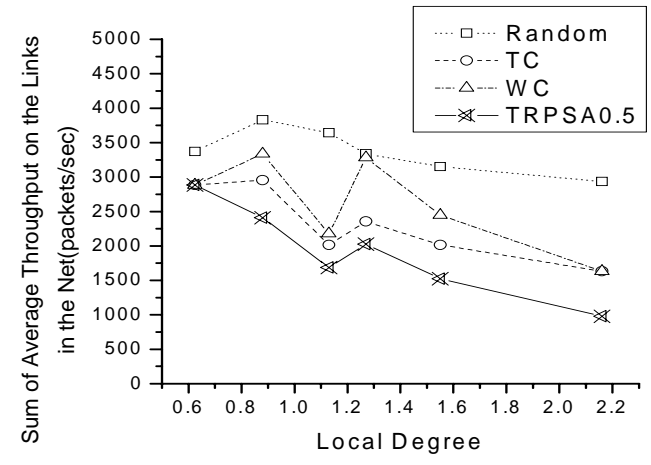

Fig.1 Multicast tree cost of different localization degrees of member distribution

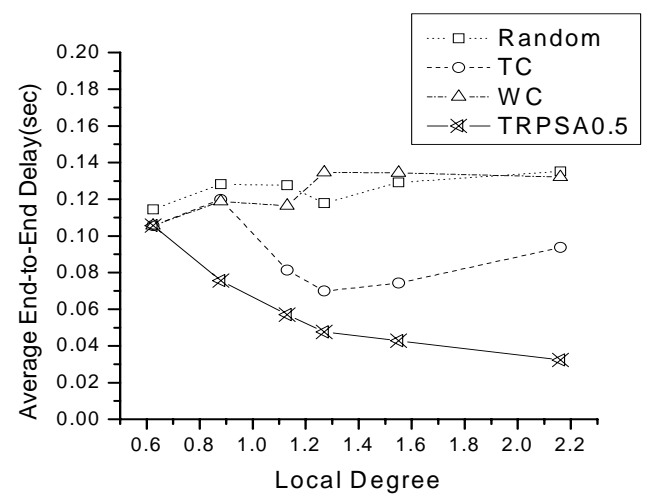

Fig. 2 The average ETE delay of different localization degrees of member distribution

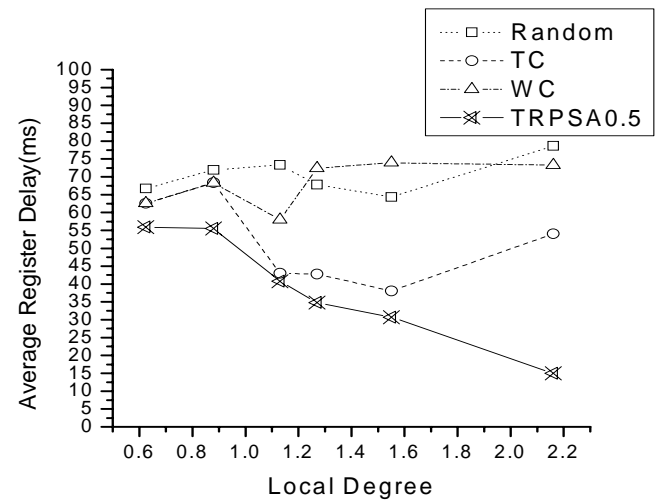

Fig.3 The average register delay of different localization degrees of member distribution

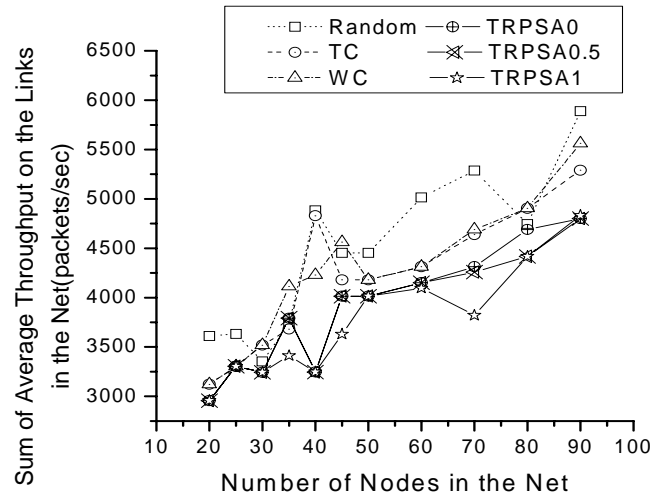

Fig. 4 Comparison of multicast tree cost in different network scales

The distribution of multicast group members in the network is very important to the result of RP selection. The ratio between the average distance of all nodes and the average distance of group members is called localization degree of group member distribution. Figures 1, 2 and 3 indicate the comparison of distribution of group members by using different algorithm. The figure after TRPSA is 
the tradeoff parameter between cost and delay. It can be seen from the figures that with the increase of localization degree of group members TRPSA performs better than other algorithms with a rapid reduction in cost, ETE delay and register delay.

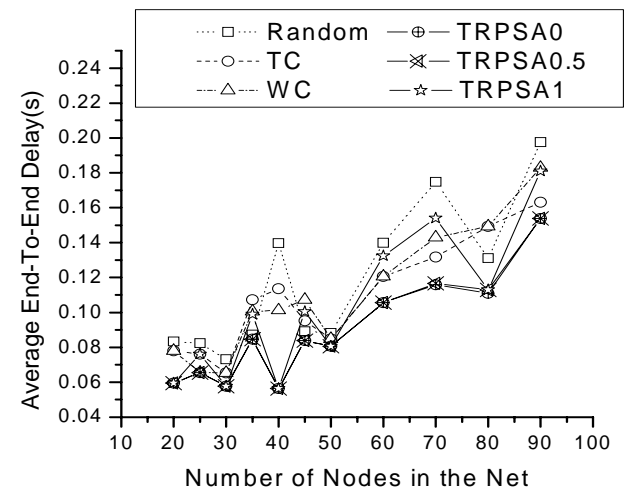

Fig. 5 The average ETE delay in different network scales

Figures 4, 5 and are the results of comparison of algorithms in different network scales. It can be seen that When $w=1$ and $w=0$, TRPSA can guarantee the minimal value of cost and average ETE delay respectively; when $w=0.5$, it can guarantee good results of cost, ETE delay and register delay. It has an overall better performance than other algorithms. The results obtained via TC and WC are similar. In most cases, they have poor performance.

\section{Conclusion}

Current RP selection algorithms cannot accurately evaluate the merits and shortcomings of multicast tree, and greedy local search algorithm is apt to local optimization. To solve these two problems, TRPSA is proposed. Simulation results indicate that this algorithm has good performance in multicast cost, ETE delay and other aspects. It is an algorithm having good expansion and practical feasibility. The parameter between cost and delay of the algorithm $w$ can be adjusted flexibly to meet different needs of application. For the application with higher demand of network resources $w$ can be adjusted bigger to reduce total network throughput rate, and for the application sensitive to delay $w$ can be adjusted smaller to reduce the average delay. The shortcoming of the algorithm is that more parameters are needed, of course, The values can be obtained through measurement in real network. Also, more information needs to be collected, including information of topology and group members. This is an inherent problem of group-based approach, which can only be solved by recurring to some network protocols like OSPF. Since the dynamic entering and leaving of group members may change the performance of multicast tree, further research will be focused on RP reselection after the dynamic entering and leaving of group members.

\section{Acknowledgements}

The study is supported by CNGI (China Next Generation Internet) Research And Experiment of Technology of Large-Scale Routing And Multicast. Grant Number CNGI-04-13-2T.

References:

[1] A. Ballardie, B. Cain, and Z. Zhang, Core Based Trees (CBT version 3) Multicast Routing. Internet Draft, 1998.

[2] D. Estrin et al, Protocol Independent Multicast-Sparse Mode (PIM-SM), Protocol Specification. RFC2362, 1998.

[3] D. Wall, Mechanisms for broadcast and selective broadcast, Ph.D. thesis, Computer Science Department, Stanford University, 1982.

[4] A.S. Carlos Oliveira*, M. P. Panos, A survey of combinatorial optimization problems in multicast routing, Computers and Operations Research, Vol. 32 No.8, 2005, pp: 1953-1981.

[5] Sung Mo Chung, Chan-Hyun Youn, Core Selection Algorithm for Multicast Routing under Multiple QoS Constraints, IEE Electronics Letters, Vol. 36, No. 4, 2000, pp: 278-279.

[6] Xiao-bo Dong, A New Algorithm for RP Selection in PIM-SM Multicast Routing, Masters Abstracts International, Vol. 41, No. 3, 2002, pp: 799-805.

[7] Dong-Lim Lee, Chan-Hyun Youn, Sang-Jin Jeong, RP Reselection Scheme for Real-time Applications in Delay-constrained Multicast Networks, ICC 2002, pp:1290 - 1294.

[8] Ritesh Mukherjee, J. William Atwood, Rendezvous Point Relocation in Protocol Independent Multicast-Sparse Mode, Telecommunication Systems, Vol. 24, No. 2/4, 2003, pp.207-220.

[9] F. Glover, Tabu Search: Part I. OSRA Journal on Computing, 1989, pp.190-206.

[10] S. Hanafi, On the Convergence of Tabu Search . Journal of Heuristics, Vol.7, 2001, pp.47-58.

[11] B. M. Waxman, Routing of multipoint connections, IEEE Journal on Selected Areas in Communications, Vol. 6, No. 9, 1998, pp.1617-1622. 